\title{
ON THE MUTAGENIC RADICAL PROPERTY
}

\author{
by G. TZINTZIS
}

(Received 20th March 1984)

\section{1.}

In their paper N. Divinsky and A. Sulinski [6] have introduced the notion of mutagenic radical property - that is, a radical property which is far removed from hereditariness-and constructed two such examples. The first is the lower radical property determined by a ring $S_{w_{0}}$ (N. Divinsky [5]) and is an almost subidempotent radical property in the sense of $F$. Szász [9], and the second is a weakly supernilpotent radical property, that is the lower radical property determined by $S_{w_{0}}$ and all nilpotent rings.

The purpose of this paper is, on the one hand, to present many other examples of mutagenic radical properties, and on the other, to introduce a more natural notion for radical classes which are far removed from hereditariness, and to examine its relation with mutagenity.

2.

Throughout this paper all rings considered will be associative. The terminology and basic results of radical theory can be found in [4], [1], [2].

Definition 1. (N. Divinsky and A. Sulinski, [6]) A radical property $\mathscr{Y}$ is said to be mutagenic if there exists a nonzero ring $R$ such that,

(1) $R=\bigcup_{a} I_{a}>\ldots>I_{a}>\ldots>I_{2}>I_{1}>0$, where $a$ ranges over some indexing set of ordinals and $I_{a}$ are strongly $\mathscr{Y}$-semisimple ideals, and

(2) $\mathscr{Y}(R) \neq 0$.

Proposition 1. Every almost subidempotent radical property $N$, with $N \cap \mathscr{B} \neq 0$, is mutagenic.

Proof. Let $R \in N \cap \mathscr{B}$ be a nonzero ring. Since $R$ is a $\mathscr{B}$-radical ring it is well known that its every nonzero homomorphic image contains a nonzero nilpotent ideal. Therefore, we can construct an ascending chain of ideals. $0<I_{1}<$ $I_{2}<\ldots<I_{a}<I_{a+1}<\ldots$, where $I_{a+1} / I_{a}$ is a nilpotent ring, for every ordinal number $a$. Evidently, since $N$ is an almost subidempotent radical property, nilpotent rings are strongly $N$-semisimple. Also, by [6], Lemma 1 , if $I_{a}$ is a strongly $N$-semisimple ring; then $I_{a+1}$ is also strongly $N$-semisimple. Finally, for a limit ordinal number $a$, we define 
$I_{a}=\bigcup_{b<a} I_{b}$ and if $I_{a}$ is a strongly $N$-semisimple ring we carry on extending the chain. Where $I_{a}$ is not a strongly $N$-semisimple ring we can take an homomorphic image which is not an $N$-semisimple ring.

Corollary 1. The almost subidempotent radical properties $I_{\mathscr{y}}$ of all idempotent $\mathscr{Y}$ radical rings (G. Tzintzis [10]), where $\mathscr{Y}=\mathscr{B}, \mathscr{L}, \mathscr{N}, \mathscr{B}_{\phi}, \mathcal{N}_{\phi}, L_{2}$ ([8]), $\Psi$ ([11]), J, J $\mathscr{P}, J_{B}, \mathscr{G}, \mathscr{N}_{g},\left(\mathscr{N}_{g}\right)_{\phi}, \mathscr{T}, \mathscr{D}, \mathscr{F}$ (N. Divinsky [4]), are mutagenic.

Corollary 2. The almost subidempotent radical property $\mathscr{X}$ (G. Tzintzis \{12]) is mutagenic.

More generally, let $M$ be an homomorphically closed class of rings which has the property that every nonzero ideal of a ring of $M$ can be mapped homomorphically onto some nonzero ring of $M$. If, as usual, we denote as $U(M)$ and $L(M)$ the upper radical property determined by $M$ and the lower radical property determined by $M$ respectively, then we have the following.

Proposition 2. $U(M)$ is mutagenic if $U(M) \cap L(M) \neq 0$ holds.

Proof. Since $M$ is homomorphically closed, it is contained in the class of strongly $U(M)$-semisimple rings. Also, if $U(M)$ is not mutagenic, then by [6] Theorem 7, the class of all strongly $U(M)$-semisimple rings must be radical, and consequently, $U(M) \cap L(M)=0$ must hold a contradiction.

Corollary 3. The radical classes $\mathscr{P}$ and $T_{U}$, where $\mathscr{P}$ is the Jenkins [7] upper radical determined by all prime rings and $T_{U}$ is the $N$. Divinsky [5] upper radical property determined by all unequivocal rings, are mutagenic.

Proof. Indeed, the ring $S_{w_{0}}$ of the example $E$ [5], is $\mathscr{P}$-radical, and simultaneously, is contained in $L(M)$, where $M$ is the class of all prime simple rings. Also, $S_{w_{0}}$ is a $T_{U^{-}}$ radical ring ([5]) and is contained in $L\left(M^{*}\right)$, where $M^{*}$ is the class of all unequivocal rings.

Definition 2. A non-trivial radical property $\mathscr{Y}$ is said to be completely non-hereditary if every nonzero $\mathscr{Y}$-radical ring contains a nonzero ideal which is not $\mathscr{Y}$-radical.

Definition 3. A non-trivial radical property $\mathscr{O}$ is said to be strongly completely nonhereditary if every nonzero $\mathscr{Y}$-radical ring contains a nonzero ideal which is strongly $\mathscr{Y}$ semisimple.

Evidently, a strongly completely non-hereditary radical property is also completely non-hereditary. An example of a completely non-hereditary radical property is the almost subidempotent radical property $\mathscr{X}$ (G. Tzintzis [12], Proposition 2.2). In general

Proposition 3. Every almost subidempotent radical property $\mathscr{Y}$, with $\mathscr{Y} \cap \mathscr{B}_{\phi}^{\prime}=0$, where $\mathscr{B}_{\phi}^{\prime}$ is the class of all hereditarily idempotent rings ([3]), is completely non-hereditary. 
Proof. Indeed, if $R$ is a nonzero $\mathscr{Y}$-radical ring, then it is not contained in $\mathscr{B}_{\phi}^{\prime}$ and consequently has a non-idempotent ideal which, evidently, is not $\mathscr{Y}$-radical.

Also, we can show that an example of a strongly completely non-hereditary radical property is the lower radical property determined by the ring $S_{w_{0}}([6]$, Example 3). Indeed, $S_{w_{0}}$ is a $\mathscr{P}$-radical ring and also hereditarily idempotent. Consequently, $L\left(\left\{S_{w_{0}}\right\}\right)$ is contained in both the classes $\mathscr{P}$ and $\mathscr{B}_{\phi}^{\prime}$ and precisely consists of all rings, every nonzero homomorphic image of which contains, as an ideal, a nonzero homomorphic image of $S_{w_{0}}$. Therefore, every nonzero $L\left(\left\{S_{w_{0}}\right\}\right)$-radical ring contains as an ideal a simple prime ring which is strongly $L\left(\left\{S_{w_{0}}\right\}\right)$-semisimple.

It is now natural to ask: What is the relation between mutagenity and (strongly) complete non-hereditariness?

Proposition 4. If $\mathscr{Y}$ is a completely non-hereditary radical property, then every nonzero Y-radical ring contains a nonzero $\mathscr{Y}$-radical ideal which is the union of an ascending chain of non-Yy-radical ideals.

Proof. Indeed, since every nonzero homomorphic image of a nonzero $\mathscr{Y}$-radical ring $R$ contains a nonzero ideal $I$ which is not $\mathscr{Y}$-radical, we can construct an ascending chain of ideals $0<I_{1}<I_{2}<\ldots<I_{n}<\ldots$, which are not $\mathscr{Y}$-radical rings. The first limit ordinal number we correspond to the union, $\bigcup_{n} I_{n}$. If $\bigcup_{n} I_{n} \neq R$ and is not a $\mathscr{Y}$-radical ring, then we carry on extending the chain.

Corollary 4. If $\mathscr{Y}$ is a strongly completely non-hereditary radical property, then every nonzero $\mathscr{Y}$-radical ring contains a nonzero ideal, some nonzero homomorphic image of which is the union of strongly $\mathscr{Y}$-semisimple ideals but is not $\mathscr{Y}$-semisimple.

Proof. Indeed, as in Proposition 4, we can construct an ascending chain of ideals $0<I_{1}<I_{2}<\ldots<I_{n}<\ldots$, which, by [6] Lemma 1, must be strongly $\mathscr{Y}$-semisimple. If for the limit ordinal number $a$ the union $\bigcup_{b<a} I_{b}$ is a strongly $\mathscr{Y}$-semisimple ring, then we carry on extending the chain. Otherwise, this union is the ideal for which we were looking.

Corollary 5. Every strongly completely non-hereditary radical property $\mathscr{Y}$ is mutagenic.

Finally, we must observe that the problem remains whether there exists an example of a completely non-hereditary radical property $\mathscr{Y}$ which is not mutagenic.

\section{REFERENCES}

1. V. A. Andrunakievic, Radicals in associative rings I, Mat. Sb. (N.S.) 44(86) (1958), 179-212 (Russian), Amer. Math. Soc. Transl. 55 (2) (1966), 95-128.

2. V. A. Andrunakievic, Radicals in associative rings II, Mat. Sb. (N.S.) 55(97) (1961), 329-346 (Russian), Amer. Math. Soc. Transl. 55(2) (1966), 129-149.

3. V. A. ANDrunakieviC, Antisimple and strongly idempotent rings, Izv. Akad. Nauk. SSSR Ser. Mat. 21 (1957), 125-144 (Russian), MR 19, 244. 
4. N. Drvinsky, Rings and Radicals (University of Toronto Press, 1964).

5. N. Divinsky, Unequivocal rings, Can. J. Math. 27(3) (1975), 679-690.

6. N. Divinsky and A. Sulinski, Radical pairs, Can. J. Math. 29(5) (1977), 1086-1091.

7. T. L. Jenkins, A maximal ideal radical class, J. Natur. Sci. and Math. 7 (1967), 191-195.

8. L. C. A. van Leeuwen and G. A. P. Heyman, A radical determined by a class of almost nilpotent rings, Acta Math. Acad. Sci. Hungar. 26 (1975), 259-262.

9. F. A. SzAsz, A radical property of rings such that every homomorphic image has no nonzero left annihilators, Math. Nachr. 48 (1971), 371-375.

10. G. Tzintzis, Almost subidempotent radicals and a generalization of a theorem of Jacobson, Hungarian Acta Math. (to appear).

11. G. Tzintzis, An upper radical property and an answer to a problem of L. C. A. van Leeuwen and G. A. P. Heyman, Acta Math. Acad. Sci. Hungar. 39(4) (1982), 381-385.

12. G. Tzintzis, An almost subidempotent radical property, to appear.

Department of Mathematics

ARISTOTLE UNIVERSITY OF THESSALONIKI

Greece 